\title{
A REPOTENCIALIZAÇÃO DE ROLAMENTOS SOB O ASPECTO DE AGREGAÇÃO DE VALOR*
}

Ricardo Rocha de Oliveira ${ }^{1}$

\section{Resumo}

A indústria Siderúrgica, nos dias atuais, vem enfrentando grandes desafios, no que tange à sua produção de metais. Com um cenário cada vez mais desafiador, (variação cambial, mercado externo, mão de obra local, custos de produção e logísticos, matériaprima), as empresas do setor de Metalurgia precisam adotar soluções cada vez mais sustentáveis e com um excelente retorno a curto e médio prazo. Mesmo sob estas condições, o mercado brasileiro não para de inovar em seus processos, principalmente a indústria automobilística, onde há vários desenvolvimentos de veículos automotores que demandam o que há de mais tecnológico e moderno, tais como ligas especiais, adoção de alumínio em sua composição, aços com maior resistência mecânica, entre outros. O Segmento de Metalurgia/Siderurgia é orientado, desta forma, a realizar as mais diversas pesquisas para a otimização de processos e de produção, através de parcerias com seus fornecedores e pesquisa de matéria-prima. Com tudo isso, se verifica que é possível aplicar novas soluções, incorporando-as aos seus processos, obtendo excelentes retornos quanto a ganhos de produtividade e redução de custos de aquisição/manutenção, sem deixar de lado o fator Sustentabilidade e a prática do "Ecologicamente Correto". Desta forma, as empresas do setor de Metalurgia/Siderurgia conseguem se destacar em seu nicho tecnológico, atendendo a demanda do mercado, apresentando excelente retorno a seus acionistas e garantindo a satisfação de seus colaboradores em todos os níveis dentro da corporação.

Palavras-chave: Pesquisa; Parceria; Sustentabilidade.

\begin{abstract}
The steel industry, nowadays, has been facing great challenges regarding its production of metals. With an increasingly challenging scenario (exchange rate variation, external market, local labor, production costs and Logistics, raw material), as Metallurgy sector companies adopt ever more sustainable solutions and with an excellent return in the short and medium time. Even under these conditions, the Brazilian market can't stop to innovate in its processes, mainly an automobile industry, where there are several developments of automotive vehicles that require the most technological and modern, such as special alloys, adoption of aluminum in its composition, Steels with greater mechanical resistance, and others.

The Steel / Metallurgy Segment are oriented in this way to carry out as many different researches for the optimization of processes and production, through partnerships with its suppliers and research of raw material. With all this, check that it is possible to apply new solutions, incorporating them as your processes, obtaining excellent returns on production gains and reduction of components acquisitions / maintenance costs, without leaving aside the factor Sustainability and practice of "Ecologically correct".

In this way, as companies in the Metallurgy/Steel sector managed to become references in its technological niche, meeting a market demand, presenting excellent returns to its shareholders and ensuring a satisfaction of its employees at all levels within the corporation.
\end{abstract}

RECONDITINING BEARINGS ADDING VALLUE ASPECTS

Keywords: Research, Partnership, Sustainability

Engenharia Mecânica,Supervisor de Engenharia, Engenharia de Aplicação e Serviços, NSK Brasil LTDA, São Paulo, São Paulo - Brasil. 


\section{INTRODUÇÃO}

O processo siderúrgico possui uma área produtiva de extrema importância, que é a área de Lingotamento Contínuo. Através de um arranjo de segmentos de rolos superiores e inferiores, o aço líquido é vazado por entre este arranjo, sob condições de velocidade e temperatura meticulosamente controladas.

Esta aplicação, demanda certas solicitações aos Mancais de Rolamentos, exigindo destes componentes alta resistência a cargas aplicadas e excelente estabilidade dimensional quando submetido a altas temperaturas.

Outra variável do processo se dá por conta da dificuldade de o lubrificante formar película de lubrificação adequada, pois, a rotação de trabalho é extremamente baixa.

Quanto aos controles preventivos destes mancais é feita uma gestão muito bem estruturada. Tem-se que cada segmento do veio do lingotamento é identificado quanto à posição e monitorado quanto à vida útil de trabalho (toneladas processadas) por componente. Estas informações preparam os mantenedores do Lingotamento quanto a eventuais falhas abruptas do componente Rolamento para possíveis substituições imediatas, evitando-se assim, maiores prejuízos no processo de solidificação do aço.

A parceria sustentável e tecnológica na gestão de ativos desta aplicação é um determinante do programa de gerenciamento de ativos - AIP, ferramenta de Engenharia de exclusividade NSK.

O programa de gerenciamento de ativos - AIP é um programa de melhoria de ativos, que combina conhecimento do ambiente de trabalho, cultura, processos e problemas do cliente final com a perícia de engenharia e inovação do fornecedor. É a oportunidade real para a obtenção de rentabilidade adicional através da melhoria da confiabilidade de uma máquina e maior conhecimento técnico.

A chave do sucesso é a interação da engenharia do fabricante de um componente com o pessoal de campo, para identificação e compreensão das questões e problemas enfrentados no dia a dia e o impacto desses problemas que afetam financeiramente o negócio.

Os benefícios do programa são respostas reais para problemas, aumento de eficiência produtiva, redução dos tempos de paradas de máquinas, aumento de rentabilidade e a manutenção da competitividade no segmento.

O enfoque do programa AIP é justamente o enfoque sustentável da parceria, que gera a redução na substituição de componentes, redução de custos com mão de obra (pois os ativos humanos são mais capacitados e utilizam os recursos de forma adequada), máquinas e processos mais confiáveis e eficientes e menores gastos energéticos.

A NSK incorpora dentro do âmbito do Programa AIP, a atividade de Repotencialização de Rolamentos, onde é possível, através de métodos e processos muito bem definidos e padronizados, uma nova utilização dos Rolamentos que a princípio, seriam descartados. 


\section{MATERIAIS E MÉTODOS}

Os métodos de aplicação deste artigo consistem na sistemática do serviço de Repotencialização, aplicado pela NSK a esta aplicação em específico.

Engloba-se esta atividade (Repotencialização) como Suporte de Engenharia, pois, a NSK aplica o que há de mais moderno no processo de Repotencialização. O Departamento de Engenharia e todo o processo de Qualidade, além dos recursos disponibilizados pelo Laboratório Brasileiro de Tecnologia (BTC) são amplamente utilizados, tudo com o objetivo de prover retorno rápido, garantia do serviço prestado, recomendações de melhorias na aplicação e redução dos custos de aquisição / manutenção.

\subsection{O AIP - Programa de Gerenciamento de Ativos}

O AIP é fundamentado em 4 grandes pilares, dos quais concentram atividades de Engenharia de Rolamentos. Agrega toda a metodologia de Análise de falhas (baseado na norma ISO 15243:2004), suportado pela norma ISO 55000: Sistema de Gestão de Ativos. Os principais Pilares do programa AIP são 4, destacados abaixo:

- Mapeamento: Atividades relacionadas ao conhecimento prévio de processo produtivo, levantamento de informações relativas a insumos, análise preditiva, manutenção preventiva, manutenção programada. Ainda, é possível utilizarse desta ferramenta para trabalhar com conversão de códigos de produtos especificamente desenvolvidos por fabricante de equipamentos. Com o conjunto destas ações, é possível mapear as oportunidades de melhorias nos equipamentos considerados com certa classe de criticidade.

- Seleção: Uma vez que o pilar do programa AIP (1) Mapeamento tenha sido implementado, o pilar (2) Seleção será utilizado para atuar nas oportunidades captadas, dos quais se têm a oportunidade de atuar com melhorias de Engenharia, reduzindo-se assim custos de manutenção e aumentando a produtividade.

- Treinamento: Pilar complementar de todo o programa AIP, pois, treinamentos sobre aplicação de Rolamentos (básico e avançado), análise de falhas e modalidade prática podem ser aplicados. Uma vez esta atividade ter sido aplicada com êxito, o potencial de otimização da aplicação de Rolamentos é aumentada consideravelmente.

- Inspeção: Pilar que contempla todo o processo de Análise de Falhas de Rolamentos. Todas as ações de Engenharia aplicadas no Pilar Seleção são fundamentalmente baseadas neste pilar. Em suma, aqui será possível determinar o êxito da aplicação de um Rolamento, quando mensurado o potencial teórico da vida de fadiga do aço rolamento.

O processo de Repotencialização passa por atividades de todos os quatro pilares. Inicialmente, o pilar (1) Mapeamento, é aplicado, de modo a identificar o fluxo de produção, quantidade de Rolamentos, temperatura de trabalho, agentes contaminantes). Baseado nestes dados, histórico da aplicação (falhas prematuras) e da necessidade de otimização dos custos de aquisição de produto, é que o pilar (2) 
Seleção é acionado, através da sub-atividade: Suporte de Engenharia. O pilar (3) Treinamento é aplicado, no âmbito de reforçar as melhores práticas de manutenção de Rolamentos Industriais, haja vista que estudos NSK apontam um alto índice de falhas de Rolamentos, atrelado a não utilização de práticas adequadas para montagem das peças. O pilar (4) Inspeção é utilizado como suporte à Repotencialização, pois, o que se objetiva aqui, não é simplesmente prover Serviço ao cliente e sim, trabalhar em conjunto com o usuário final, identificando problemas relativos à aplicação, bem como as melhores recomendações.

\subsection{Processo de Repotencialização}

\subsection{1) Qual o objetivo da Repotencialização?}

O objetivo da Repotencialização é de tornar possível uma nova fase de operação a Rolamentos que já operaram por determinado tempo. O êxito deste processo consiste na utilização de vários procedimentos, tais como: limpeza, processo de retífica primária, polimento, controle da qualidade de acordo ao processo adotado para Rolamentos Novos NSK, Aplicação de Protetivo (anti-oxidante) e reembalagem. Este processo renovador de vida restaura os rolamentos, tornando possível seu desempenho sob as mesmas características de um Rolamento novo.

\subsection{2) Quando Repotencializar?}

As verificações de manutenção de rotina podem indicar sinais de fadiga do Rolamento. Além disso, conhecer o histórico, o desempenho e o ambiente operacional fornecerão indicações quanto à condição do rolamento. Nem todos os rolamentos se beneficiarão das propriedades regeneradoras de vida do recondicionamento. Alguns rolamentos podem estar muito danificados, exigindo a compra de um novo rolamento.

\subsection{3) Por que Repotencializar?}

Muitas vezes, um rolamento pode ser repotencializado e colocado de volta em operação, estendendo assim, sua vida útil. Desta forma, minimizam-se impactos causados por custos elevados repentinos e longos prazos de aquisição para um novo rolamento. Os rolamentos repotencializados que não são devolvidos ao serviço imediatamente, mas armazenados para serviço posterior, podem reduzir os custos de inventário. Recondicionamento pode custar uma fração do tempo e dinheiro em comparação com o novo. A qualidade dos rolamentos repotencializados é suportada pelos processos de repotencialização. Engenheiros / Técnicos especializados e equipamentos de alta qualidade permitem uma sobrevida no rolamento. As especificações originais são usadas para garantir a qualidade, funcionalidade e desempenho do rolamento. A repotencialização é um meio oportuno, confiável e econômico de manter a produtividade e reduzir o tempo de inatividade.

\subsection{Processo de Checagens}

A Repotencialização é suportada por diversos pontos de checagem, desde o recebimento do material até o fim do processo. Já no início, um processo rigoroso aplicado é o da Inspeção Preliminar, que tem como objetivo fazer uma inspeção criteriosa na peça e verificar a aplicabilidade da repotencialização. Em alguns casos, faz-se necessária inspeção "in loco" da Engenharia de Aplicação e Serviços da NSK. Uma vez que não seja identificada nenhuma anomalia no Rolamento que impeça o processo de Repotencialização (Substituição de Elementos Rolantes ou outro componente, Escamamento de Pista, micro-trinca/trinca, fratura de anéis e/ou componentes, severa corrosão nos elementos do Rolamento), o Rolamento é 
aprovado para a efetivação do processo de Repotencialização. O Fluxo macro do processo é apresentado abaixo:

- Recebimento e identificação do rolamento (criação de rastreio sistêmico dos itens);

- Inspeção visual inicial;

- Processo de lavagem e limpeza;

- Inspeção visual;

- Análise dimensional, incluindo folga e ovalização;

- Desmontagem do rolamento;

- Aplicação do processo RPT (retífica, polimento, ajustes);

- Verificação e inspeção visual;

- Inspeção por Partículas Magnéticas (Magnaflux);

- Montagem do rolamento;

- Gravação especifica;

- Inspeção de Qualidade incluindo laudo técnico;

- Aplicação de óleo protetivo;

- Embalagem.

Uma vez concluído o processo, toda a rastreabilidade do item (avaliação dimensional antes e após a repotencialização, folga radial, relatório fotográfico) é compilada em Relatório Técnico, criando histórico de cada peça repotencializada.

\subsection{Normatização do Processo}

Por se tratar de uma empresa multinacional, principalmente com reconhecimento no mercado brasileiro, é de extrema importância que todo o processo seja incorporado às práticas já estipuladas pelas certificações ISO9001, ISO14001 e ISO TS16949. Desta forma, todo o processo de Repotencialização é controlado por normas de procedimento interno (baseado nas normas acima estipuladas), com respectivas checagens periódicas e processos de auditoria quando necessário. Desde o recebimento do Rolamento até o retorno ao usuário final, há um procedimento interno (documento NSK), que contém a seguinte estrutura: (1) Qual o objetivo da etapa; (2) Resultados esperados; (3) Parâmetros da etapa de acordo com 0 processo fabril de Rolamentos novos NSK (normas ISO e normas NES).

\section{RESULTADOS E DISCUSSÃO}

Os resultados dos métodos propostos compreendem um período mapeado de 12 meses (1,0 ano).

Ao todo, a NSK recebeu deste cliente, um total de mais de 3000 peças que, a princípio seriam descartadas caso a Repotencialização não fosse implementada.

Após todo o processo abrangido nos tópicos 2.2 e 2.3 terem sido aplicados em sua totalidade, tivemos um excelente retorno, onde foi possível Repotencializar um total de mais de 1200 peças, onde as mesmas retornaram ao processo e apresentam até o momento excelentes resultados de performance operacional. Segue abaixo 0 resultado: 
Tabela 1. Resumo do Trabalho

\section{Ano Peças Segregadas Peças Aprovadas Peças ${ }^{1}$ Reprovadas}

\begin{tabular}{llll}
\hline 2016 & 3287 & $1281(39 \%)$ & $2026(61 \%)$
\end{tabular}

'Dentre os Rolamentos reprovados, temos as seguintes avarias identificadas na inspeção preliminar:

\begin{tabular}{|c|c|}
\hline Avarias & Quantidade \\
\hline Folga Excessiva (Desgaste Excessivo) & 851 \\
\hline Escamamento Pista / Rolos & 415 \\
\hline Oxidação (geral) & 365 \\
\hline Ovalização & 328 \\
\hline Pitting & 233 \\
\hline Trincas & 138 \\
\hline
\end{tabular}

Tabela 2. Avarias nos Rolamentos

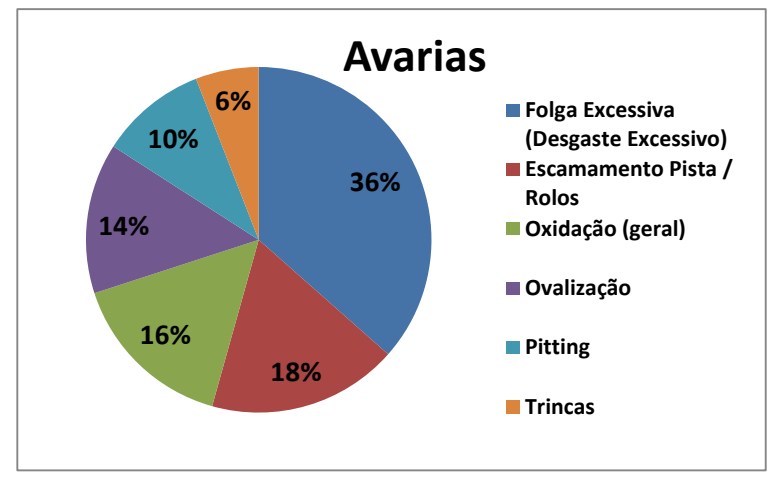

Gráfico 1. Avarias nos Rolamentos

\section{1) Tecnologia SWR ${ }^{\mathrm{TM}} N S K$}

Analisando o cenário acima, a NSK, em parceria com o cliente, está atuando na aplicação de uma nova tecnologia de Rolamentos, o qual foi desenvolvida para combater falhas ocasionadas aos Rolamentos advindas de Desgaste excessivo e consequente escamamento de pista/elementos rolantes (comum a esta aplicação). As condições adversas de operação produzem efeitos de falha acelerada aos Rolamentos, onde o componente é mais solicitado, inevitavelmente. Desta forma, a NSK está estudando a aplicação de um Rolamento com uma composição de seu aço com maior resistência ao desgaste, o que permite atingir uma maior vida útil de até 5x mais, quando comparado com o aço Rolamento convencional (SAE 52100). 


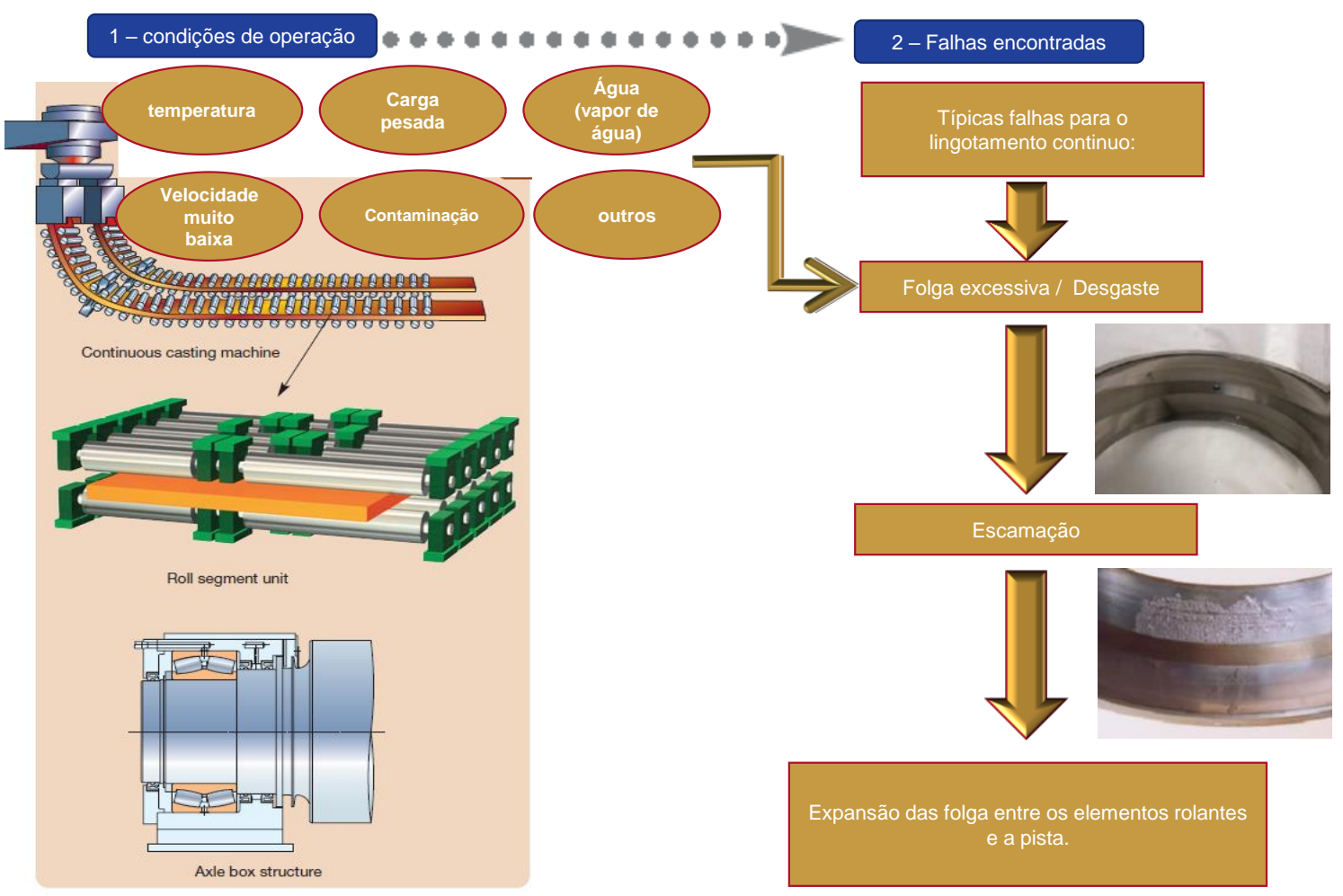

Rolamentos de rolos esféricos da serie SWR.

- Melhor resistência ao desgaste $\rightarrow$ Três vezes em relação ao aço de rolamento AISI 52100

- Melhoria da propriedade de vida de descascamento $5 x$ maior, em relação ao aço de

AISI 52100

- Maior resistência do núcleo do material

(Prevenção de danos por fissuras) $\rightarrow 5 x$ maior em relação ao aço de rolamento AISI 52100

Fig.1 Esquema de falha dos Rolamentos e Nova Tecnologia aplicada

\section{2) Ganhos obtidos com a Repotencialização}

Os ganhos obtidos com o projeto são de alta relevância para o cliente final. A seguir, é apontado o cenário antes e após a Repotencialização:

Tab. 3: Extratificação dos Ganhos (R\$)

\begin{tabular}{|cccc|}
\hline Ano & Peças Segregadas & $\begin{array}{c}\text { Peças } \\
\text { Efetivamente } \\
\text { Reprovadas }\end{array}$ & $\begin{array}{c}\text { Ganho com } \\
\text { Repotencialização } \\
\text { NSK }\end{array}$ \\
\hline 2016 & $\mathrm{R} \$ 3,4$ Mio & $\mathrm{R} \$ 2,9$ Mio $(85 \%)$ & $\mathrm{R} \$ 0,43$ Mio $(15 \%)$ \\
\hline
\end{tabular}

Podemos observar que o cliente conseguiu reduzir $15 \%$ de seu custo inicial com aquisição de Rolamentos novos, ocasionando uma economia final de quase $\mathbf{k R \mathbf { S }}$ $\underline{\mathbf{4 3 0}, 00}$ em 1 ano de atendimento dedicado da equipe de Engenharia de Aplicação e Serviços da NSK. 


\section{CONCLUSÃO}

Estes são apenas parte do ganho mensurável com a aplicação deste trabalho. Por exemplo, temos os ganhos correlatos à sustentabilidade do processo, onde um rolamento repotencializado requer até $90 \%$ menos energia do que a produção de um novo, promovendo inclusive, uma significativa redução nos níveis de emissão de CO2 na atmosfera terrestre. A utilização de insumos utilizados no processo de fabricação de um rolamento é consideravelmente reduzida, por não ser necessária a adoção de certas etapas do processo fabril, como por exemplo, tornearia, tratamento térmico, eliminação de etapas de usinagem por retificação. Ainda, descarte de óleo solúvel (usinagem) é reduzido consideravelmente, pois, o processo de usinagem de uma peça nova requer muito mais refrigeração e lubricidade do que um Rolamento necessita para ser repotencializado.

A parceria sustentável e tecnológica entre o fornecedor e o cliente final baseada no programa de gerenciamento de ativos AIP além de gerar respostas mais eficazes na solução de problemas, promove a cultura de gestão dos ativos da empresa (equipamentos e colaboradores), reduz a troca de componentes, reduz o consumo energético, torna as máquinas e processos mais eficientes, aumenta a confiabilidade das máquinas e principalmente promove a utilização de recursos da forma mais adequada possível.

\section{Agradecimentos}

Agradeço em primeiro lugar a Deus. Agradeço também aos gestores e colaboradores de nosso cliente e também a alta direção e gerentes da NSK pelo envolvimento nos projetos e atividades desta parceria sustentável e de sucesso.

\section{REFERÊNCIAS}

1 Catálogo Geral de Rolamentos NSK: Autor: NSK LTDA

2 AIP: Programa de Gerenciamento de Ativos: Autor: NSK LTDA.

3 ISO 15243:2004 - Rolling Bearings - Damage and Failures - Terms, characteristics and causes: Autor: ISO

4 ABNT NBR ISO 55000, Gestão de Ativos - Fundamentos, princípios e terminologia: Autor: ISO 